\title{
Comments on the Rate of Convergence between Mann and Ishikawa Iterations Applied to Zamfirescu Operators
}

\author{
Yuan Qing ${ }^{1}$ and B. E. Rhoades ${ }^{2}$ \\ ${ }^{1}$ Department of Mathematics, Hangzhou Normal University, Hangzhou 310036, Zhejiang, China \\ ${ }^{2}$ Department of Mathematics, Indiana University, Bloomington, IN 47405-7106, USA
}

Correspondence should be addressed to Yuan Qing, yuanqingbuaa@hotmail.com

Received 16 November 2007; Revised 8 February 2008; Accepted 13 March 2008

In the work of Babu and Vara Prasad (2006), the claim is made that Mann iteration converges faster than Ishikawa iteration when applied to Zamfirescu operators. We provide an example to demonstrate that this claim is false.

Copyright (C) 2008 Y. Qing and B. E. Rhoades. This is an open access article distributed under the Creative Commons Attribution License, which permits unrestricted use, distribution, and reproduction in any medium, provided the original work is properly cited.

We begin with some definitions.

Definition 1. Suppose that $\left\{a_{n}\right\}$ and $\left\{b_{n}\right\}$ are two real convergent sequences with limits $a$ and $b$, respectively. Then $\left\{a_{n}\right\}$ is said to converge faster than $\left\{b_{n}\right\}$ if

$$
\lim \left|\frac{a_{n}-a}{b_{n}-b}\right|=0 \text {. }
$$

Definition 2. Let $(X, d)$ be a complete metric space, and $T: X \rightarrow X$ a map for which there exist real numbers, $a, b$, and $c$ satisfying $0<a<1,0<b, c<1 / 2$ such that for each pair $x, y \in X$, at least one of the following is true:

(1) $d(T x, T y) \leq a d(x, y)$;

(2) $d(T x, T y) \leq b[d(x, T x)+d(y, T y)]$

(3) $d(T x, T y) \leq c[d(x, T y)+d(y, T x)]$.

Definition 3. Let $E$ denote an arbitrary Banach space, $T$, a self-map of $E$. The sequence $\left\{x_{n}\right\}$ defined by

$$
x_{0} \in E, \quad x_{n+1}=\left(1-\alpha_{n}\right) x_{n}+\alpha_{n} T x_{n}, \quad n=0,1,2, \ldots,
$$

where $0 \leq a_{n}<1$ for $n=1,2, \ldots$, is called Mann iteration, and will be denoted by $M\left(x_{0}, \alpha_{n}, T\right)$. 
The sequence $\left\{y_{n}\right\}$ defined by

$$
\begin{gathered}
y_{0} \in E, \quad y_{n+1}=\left(1-\alpha_{n}\right) y_{n}+\alpha_{n} T z_{n}, \\
z_{n}=\left(1-\beta_{n}\right) y_{n}+\beta_{n} T y_{n}, \quad n=0,1,2, \ldots,
\end{gathered}
$$

where $0 \leq \alpha_{n}, \beta_{n} \leq 1$ for $n=1,2, \ldots$, is commonly called Ishikawa iteration, and will be denoted by $I\left(y_{0}, \alpha_{n}, \beta_{n}, T\right)$.

The following appears in [1, Theorem 2.1].

Theorem 4. Let $E$ be an arbitrary Banach space, $K$ a closed convex subset of $E, T$ a Zamfirescu operator, $0 \leq \alpha_{n}, \beta_{n} \leq 1$, and $\sum \alpha_{n}=\infty$. Then Mann iteration $M\left(x_{0}, \alpha_{n}, T\right)$ converges faster than Ishikawa iteration $I\left(y_{0}, \alpha_{n}, \beta_{n}, T\right)$ to the fixed point $x^{*}$ of $T$, provided that $x_{0}=y_{0} \in K$.

Let $T$ be a nondecreasing continuous self-map of $[0,1]$ with $p$ a fixed point of $T$. It was shown in [2, Theorem 7], that $\left|y_{n+1}-p\right| \leq\left|x_{n+1}-p\right|$ for each $n=1,2, \ldots$. Therefore, the condition

$$
\lim \left|\frac{x_{n+1}-p}{y_{n+1}-p}\right|=0
$$

is impossible for any Zamfirescu operator on $[0,1]$. The error is caused by the inconsistent in [1, Definiton 1.3] (see also [3]).

In fact, we will give an example satisfying the condition of [1, Theorem 2.1] such that the Ishikawa iteration converges faster than the Mann iteration.

Example 5. Suppose

$$
\begin{gathered}
T:[0,1] \longrightarrow[0,1]:=\frac{1}{2} x, \\
\alpha_{n}=\beta_{n}=0, \quad n=1,2,3, \ldots, 15 ; \quad \alpha_{n}=\beta_{n}=\frac{4}{\sqrt{n}}, \quad n \geq 16 .
\end{gathered}
$$

It is clear that $T$ is a Zamfirescu operator with a unique fixed point $x^{*}=0$. And it is easy to see that $T, \alpha_{n}, \beta_{n}$ satisfy all the conditions of Theorem 4 . But we show that the Ishikawa iteration $I\left(x_{0}, \alpha_{n}, \beta_{n}, T\right)$ converges faster than the Mann iteration $M\left(x_{0}, \alpha_{n}, T\right)$.

Since $\alpha_{n}=\beta_{n}=0, n=1,2,3, \ldots, 15$, so

$$
x_{n}=y_{n}=x_{0}, \quad n=1,2,3, \ldots, 16
$$

Suppose $x_{0} \neq 0$. The Mann iteration $M\left(x_{0}, \alpha_{n}, T\right)$ is

$$
\begin{aligned}
x_{n+1} & =\left(1-\alpha_{n}\right) x_{n}+\alpha_{n} T x_{n}=\left(1-\frac{4}{\sqrt{n}}\right) x_{n}+\frac{4}{\sqrt{n}} \cdot \frac{1}{2} x_{n} \\
& =\left(1-\frac{2}{\sqrt{n}}\right) x_{n}=\cdots=\prod_{i=16}^{n}\left(1-\frac{2}{\sqrt{i}}\right) x_{16}=\prod_{i=16}^{n}\left(1-\frac{2}{\sqrt{i}}\right) x_{0} .
\end{aligned}
$$


The Ishikawa iteration $I\left(x_{0}, \alpha_{n}, \beta_{n}, T\right)$ is

$$
\begin{aligned}
y_{n+1} & =\left(1-\alpha_{n}\right) y_{n}+\alpha_{n} T\left(\left(1-\beta_{n}\right) y_{n}+\beta_{n} T y_{n}\right) \\
& =\left(1-\frac{4}{\sqrt{n}}\right) y_{n}+\frac{4}{\sqrt{n}} \cdot \frac{1}{2}\left(\left(1-\frac{4}{\sqrt{n}}\right) y_{n}+\frac{4}{\sqrt{n}} \cdot \frac{1}{2} y_{n}\right) \\
& =\left(1-\frac{2}{\sqrt{n}}-\frac{4}{n}\right) y_{n}=\cdots=\prod_{i=16}^{n}\left(1-\frac{2}{\sqrt{i}}-\frac{4}{i}\right) y_{16}=\prod_{i=16}^{n}\left(1-\frac{2}{\sqrt{i}}-\frac{4}{i}\right) x_{0} .
\end{aligned}
$$

So,

$$
\left|\frac{y_{n+1}-0}{x_{n+1}-0}\right|=\frac{\prod_{i=16}^{n}(1-2 / \sqrt{i}-4 / i) x_{0}}{\prod_{i=16}^{n}(1-2 / \sqrt{i}) x_{0}}=\prod_{i=16}^{n}\left(1-\frac{4 / i}{1-2 / \sqrt{i}}\right)=\prod_{i=16}^{n}\left(1-\frac{4}{i-2 \sqrt{i}}\right) .
$$

But

$$
\begin{aligned}
0 & \leq \lim _{n \rightarrow \infty} \prod_{i=16}^{n}\left(1-\frac{4}{i-2 \sqrt{i}}\right) \leq \lim _{n \rightarrow \infty} \prod_{i=16}^{n}\left(1-\frac{4}{i}\right) \leq \lim _{n \rightarrow \infty} \prod_{i=16}^{n}\left(1-\frac{1}{i}\right) \\
& =\lim _{n \rightarrow \infty}\left(\frac{15}{16} \cdot \frac{16}{17} \cdot \frac{17}{18} \cdots \frac{n-1}{n}\right)=\lim _{n \rightarrow \infty} \frac{15}{n}=0 .
\end{aligned}
$$

Hence,

$$
\lim _{n \rightarrow \infty}\left|\frac{y_{n+1}-0}{x_{n+1}-0}\right|=\lim _{n \rightarrow \infty} \prod_{i=16}^{n}\left(1-\frac{4}{i-2 \sqrt{i}}\right)=0
$$

That is the Ishikawa iteration converges faster than the Mann iteration to the fixed point $x^{*}=0$ of $T$. So Theorem 4 is inconsistent.

\section{References}

[1] G. V. R. Babu and K. N. V. V. Vara Prasad, "Mann iteration converges faster than Ishikawa iteration for the class of Zamfirescu operators," Fixed Point Theory and Applications, vol. 2006, Article ID 49615, 6 pages, 2006.

[2] B. E. Rhoades, "Comments on two fixed point iteration methods," Journal of Mathematical Analysis and Applications, vol. 56, no. 3, pp. 741-750, 1976.

[3] O. Popescu, "Picard iteration converges faster than Mann iteration for a class of quasi-contractive operators," Mathematical Communications, vol. 12, no. 2, pp. 195-202, 2007. 